\title{
Analytical Modeling of High-Frequency Winding Loss in Round-Wire Toroidal Inductors
}

\author{
David Elizondo, Ernesto L. Barrios, Pablo Sanchis, Alfredo Ursua \\ Dept. of Electrical, Electronic and Communications Engineering \\ Public University of Navarre \\ Pamplona, Spain \\ david.elizondo@unavarra.es
}

\begin{abstract}
Toroidal inductors are present in many different industrial applications, thus, still receive researchers' attention. AC winding loss in these inductors have become a major issue in the design process, since switching frequency is being continuously increased in power electronic converters. Finite element analysis software or analytical models such as Dowell's are the main existing alternatives for their calculation. However, the first one employs too much time if different designs are to be evaluated and the second one lacks accuracy when applied to toroidal inductor windings. Looking for an alternative that overcomes these drawbacks, this paper proposes an accurate, easy-to-use analytical model, specifically formulated for calculating high-frequency winding loss in round-wire toroidal inductors.
\end{abstract}

Keywords - toroidal inductor, resistance factor, winding loss, powder core, inductor power losses, analytical model.

\section{INTRODUCTION}

Toroidal inductors have appeared in many recent papers [1]-[5]. The main reasons why toroidal inductors have been drawing such attention are their characteristics, concerning the core geometry - thanks to the toroidal shape, the magnetic flux is well contained [6] -. Moreover, toroidal cores offer a large choice of magnetic materials, e.g. ferrites, amorphous, nanocrystalline and iron powders [3]. Ungapped toroidal inductors (including cores with distributed air gap) are present in a wide variety of industrial applications. Among those applications, where inductors perform a major role, are filters [3], [4], [7], DC-link inductors in current source inverters [5] and resonant tanks [8]. As can be observed, particularly lowpermeability toroidal inductors are implemented in power applications $(>1 \mathrm{~kW})$. Toroidal powder cores stand out due to their interesting properties, including high saturation flux density, high Curie temperature, soft-saturation property and low price [1], [9].

The development of wide bandgap (WBG) power semiconductor switches is leading to higher switching frequencies in power electronic converters [5]. Regarding power applications, the switching frequency has increased as well. However, it is typically limited below $100 \mathrm{kHz}$. On the one hand, this trend of increasing the switching frequency allows to reduce inductive components size, which state as elements that increase the system cost and volume [1]. On the other hand, it means greater impact of AC copper loss or winding loss. During the inductor design stage, not only DC, but also AC copper loss must be accurately calculated, due to the inductors impact on volume, cost and cooling requirements. It has already been stated how relevant toroidal inductors are, especially powder core ones, thus becoming clear that their proper design is a major issue. Winding loss

This work was supported by the Spanish State Research Agency (AEI) under grants PID2019-110956RB-I00 /AEI/ 10.13039 and DPI-201680641-R. can be accurately known using finite element analysis (FEA) software. However, considering that great number of iterations is needed during the design stage, the computational and time costs are non-affordable. Therefore, analytical models that calculates winding or AC copper loss, such as the one developed by Dowell [10], may be useful for inductor designers.

Dowell's model concluded with the next formula, as presented in [11], which enables the calculation of the resistance factor $\left(F_{R}\right)$, that is, the $\mathrm{AC}$ to $\mathrm{DC}$ resistance ratio:

$$
\begin{gathered}
F_{R}=\frac{R_{a c}}{R_{d c}}=\Delta \cdot\left[\frac{\sinh (2 \Delta)+\sin (2 \Delta)}{\cosh (2 \Delta)-\cos (2 \Delta)}+\right. \\
\left.+\frac{2\left(m^{2}-1\right)}{3} \cdot \frac{\sinh (\Delta)-\sin (\Delta)}{\cosh (\Delta)+\cos (\Delta)}\right],
\end{gathered}
$$

where $m$ is the number of layers and $\Delta$ is the ratio between $d$ (the thickness of the winding, assuming square wires or foils) and $\delta$ (the skin depth):

$$
\delta=\frac{1}{\sqrt{\pi \cdot \mu_{0} \cdot f \cdot \sigma}}
$$

where $\mu_{0}$ is the vacuum permeability, $f$ is the frequency and $\sigma$ is the wire electrical conductivity. If Dowell's formula is applied to toroidal inductors, winding loss is significantly overestimated. Hence, designers may consider higher inductor losses than actual ones, thus, resulting in bulkier and more expensive designs. The main reason for this overestimation is that Dowell's formula, originally meant for square-window transformers [10], assumes one-dimensional magnetic field. However, the actual magnetic field in toroidal inductors is two-dimensional and the winding loss is strongly dependent on geometry. Despite this fact, Dowell's formula is still the most commonly used model for the calculation of toroidal inductor winding loss [2], [5], [12].

This paper proposes a new model to calculate $\mathrm{AC}$ winding loss in round-wire toroidal inductors with no concentrated air gap (such as the one depicted in Fig. 1), hence valid for ungapped solid cores (e.g. ferrites), as well as for powder cores and air cores. In the proposed model, each layer of the original round-wire toroidal winding is treated as a straight foil through two specific geometrical transformations, thus resulting a winding arrangement with a 1-D magnetic field. Then, power flux densities in the inner and outer sides of the layer are calculated by means of the Poynting vector and power loss per layer is obtained by adding them and calculating the real part. Finally, the winding AC resistance is computed and divided by the DC resistance, leading to a closed-form expression for the resistance factor. The proposed formula is demonstrated to be easy-to-use and to significantly reduce the estimation error, compared to existing model, thus, overcoming the main disadvantages of both FEA software and Dowell's formula. 


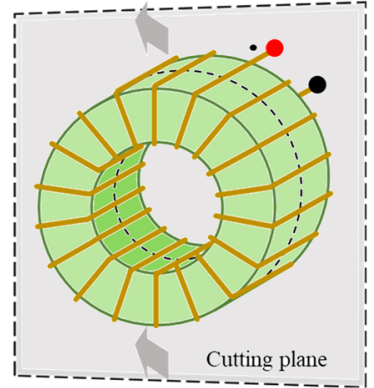

Fig. 1. 3D representation of a round-wire toroidal inductor.

\section{Proposed Model FOR TOROIDAL WINDING Loss}

As a consequence of AC current circulating through the winding, each of its turns generates a variable magnetic field. Therefore, each turn is affected not only by the surrounding turns (proximity effect), but also by itself (skin effect). Proximity and skin effects together result in AC loss in the inductor winding. Taking this into account, the proposed model starts studying the magnetic field distribution in roundwire toroidal inductors.

Inductor core shape, wire section and winding arrangement determine the magnetic field distribution in the coil. Hence, it becomes clear that core and winding geometry plays a paramount role in winding loss. In order to accurately obtain winding loss, the key geometrical parameters must be defined in the first place. Considering the case studied in this paper - the round-wire toroidal inductor - the core geometry is defined by both the inner $(I D)$ and the outer $(O D)$ diameters, as presented in Fig. 2. Regarding the solid round wire, its diameter $(2 R)$ is the parameter of interest.

Looking at the toroidal inductor cross-section in Fig. 3, it is easy to see how the winding is built: it is placed in a concentric way, starting from the layer which is closest to the magnetic core (layer 1) and finishing in layer $m$, being layer $n$ any between the first and the last layers. The result is a 'doughnut-shaped' winding, characterized for being hollow in the centre. It is important to notice that each turn goes over the outer side of the core (green in Fig. 2 and Fig. 3) and continues through its inner side (red in Fig. 2 and Fig. 3), then starting the next turn.

As the core inner window has a limited space, $I D$ is the parameter - along with the wire diameter - that sets the maximum number of turns, i.e. the larger $I D$ and the smaller $2 R$, the higher the maximum number of turns in the $n t h$ layer, $b_{n, \max }$, which can be calculated as:

$$
b_{n, \max }=\pi \cdot(A-2 \cdot(n-1)-1),
$$

where $A$ is a geometrical factor, specific for round-wire toroidal inductors, which plays a major role in the proposed model, since defines the relationship between the main geometrical parameters of the inductor: inner diameter $(I D)$ and wire diameter $(2 R)$. This factor will appear in the final expression for the resistance factor and can be expressed as:

$$
A=I D / 2 R \text {. }
$$

The actual number of turns per layer $\left(b_{n}\right)$ may be equal or less than $b_{n, \max }$, but never greater. If the winding is built so $b_{n}<$ $b_{n \text {, max }}$, there is a bigger separation between turns, that is, a higher porosity. Summing $b_{n}$ for all layers, the total number of turns in the inductor is obtained:

$$
b=\sum_{n=1}^{m} b_{n}
$$

Once the main geometrical features are defined, the model determines the magnetic field distribution in the round-wire toroidal inductor.

\section{A. Magnetic field intensity per layer}

The magnetic field in a toroidal inductor appears in circular, concentric lines, as shown in Fig. 3, as long as there is no sudden change in the permeability along the magnetic path. Thus, the proposed model assumes inductors with ungapped, powder or air cores.

According to Ampere's law, in the empty space of the inner window there is no magnetic field because there is no current circulating inside any closed trajectory. On the other hand, a closed trajectory inside the core (see Fig. 3; dark blue, dashed circumference) contains inside all $b$ turns, with a current $I$ in the same direction. Hence, again applying Ampere's law, the maximum magnetic field intensity exists in the core. As represented in Fig. 3, in layer $n$ the magnetic field intensity in the inner side is $H_{n, i}^{i}$ just before the $n t h$ layer and $H_{n, o}^{i}$ just after it:

$$
H_{n, i}^{i}=\frac{x_{n}}{\omega_{n, i}^{i}} \cdot I, \quad H_{n, o}^{i}=\frac{x_{n}+b_{n}}{\omega_{n, o}^{i}} \cdot I .
$$

Regarding the outer side, the magnetic field intensity in the $n t h$ layer is defined in the same way as:

$$
H_{n, i}^{o}=\frac{x_{n}+b_{n}}{\omega_{n, i}^{o}} \cdot I, \quad H_{n, o}^{o}=\frac{x_{n}}{\omega_{n, o}^{o}} \cdot I .
$$

For both (6) and (7), the number of turns which contribute to the magnetic field intensity in layer $n$, apart from layer $n$ itself, is defined as:

$$
x_{n}=\sum_{j=n+1}^{m} b_{j},
$$

and $\omega_{n, i}^{i}, \omega_{n, o}^{i}, \omega_{n, i}^{o}, \omega_{n, o}^{o}$ are the concentric circumferences of the trajectories in which $H_{n, i}^{i}, H_{n, o}^{i}, H_{n, i}^{o}, H_{n, o}^{o}$ are calculated, respectively. Taking into account that usually $I D \gg 2 R$, an acceptable approximation is to consider that $\omega_{n, i}^{i}=\omega_{n, o}^{i}=\omega_{n}^{i}$ and $\omega_{n, i}^{o}=\omega_{n, o}^{o}=\omega_{n}^{o}$ [13]. The $n t h$ layer heights, $\omega_{n}^{i}$ and $\omega_{n}^{o}$, are the virtual circumferences along which the $b_{n}$ turns are set in the inner and the outer sides, respectively, and are expressed as follows:

$$
\begin{gathered}
\omega_{n}^{i}=\pi \cdot(I D-4 R \cdot(n-1)-2 R), \\
\omega_{n}^{o}=\pi \cdot(O D+4 R \cdot n-2 R) .
\end{gathered}
$$

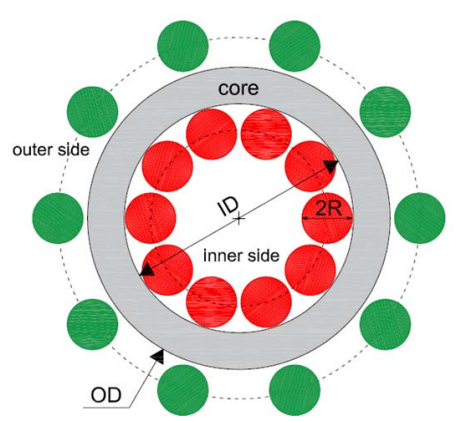

Fig. 2. Basic geometrical parameters in toroidal inductors. 


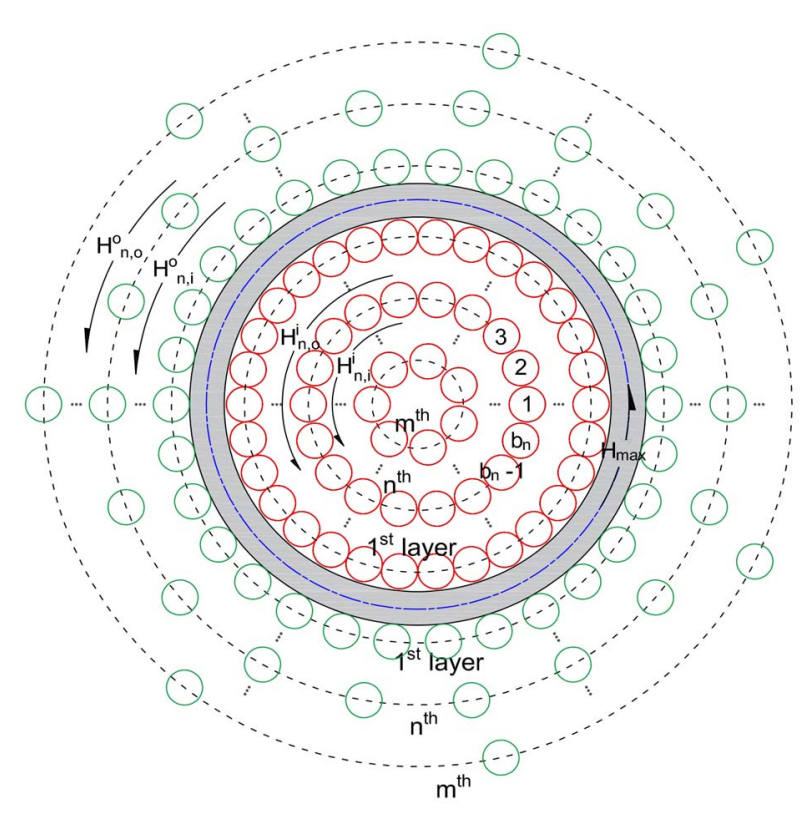

Fig. 3. Winding setup and magnetic field intensity in m-layer, round-wire toroidal inductor.

Therefore, the magnetic field intensities are now calculated considering the layer heights (in black, dashed lines in Fig. 3) and become:

$$
\begin{aligned}
& H_{n, i}^{i}=\frac{x_{n}}{\omega_{n}^{i}} \cdot I, \quad H_{n, o}^{i}=\frac{x_{n}+b_{n}}{\omega_{n}^{i}} \cdot I ; \\
& H_{n, i}^{o}=\frac{x_{n}+b_{n}}{\omega_{n}^{o}} \cdot I, \quad H_{n, o}^{o}=\frac{x_{n}}{\omega_{n}^{o}} \cdot I .
\end{aligned}
$$

The necessity of splitting the winding into two (inner and outer sides) becomes clear in (9), due to the geometrical particularities of toroidal cores. This differentiation has been done as well for the magnetic field in (10). As a result, the winding loss in the inner and outer sides have also major differences, showing that an accurate model must include the toroidal core features.

\section{B. Equivalent winding}

As depicted in Fig. 3, in the toroidal winding the magnetic field distribution is two-dimensional. However, the doughnutshaped winding - depicted in Fig. 4 (a) - can be unfolded, resulting in the arrangement of Fig. 4 (b), and the magnetic field can be treated as a one-dimensional field [13]. That is, the 2-D problem becomes a 1-D problem. Then, the roundwire winding layers are replaced by foil conductors with equivalent thickness $(d)$ and conductivity $\left(\sigma_{\omega}\right)$. For this purpose, only two transformations are needed. Firstly, as treated in [11], the round-section wire with diameter being $2 R$ is replaced by square-section wire, in such a way that the same conductor area is ensured - see Fig. 4 (c) -. This equivalent thickness is expressed as:

$$
d^{2}=\pi \cdot R^{2} \Rightarrow d=\sqrt{\pi} \cdot R .
$$

Secondly, each layer (height $\omega_{n}^{i}$ or $\omega_{n}^{o}$, depending on the side, thickness $d$ and $b_{n}$ conductors) is replaced by a foil, adapting the transformation in [11] to this case. In order to keep the DC resistance unaltered, an equivalent conductivity $\left(\sigma_{\omega}\right)$ for the foil is obtained through by modifying the conductivity $(\sigma)$ :

$$
\begin{gathered}
\sigma \cdot b_{n} \cdot \pi \cdot R^{2}=\sigma_{\omega} \cdot \omega_{n} \cdot d \Rightarrow \\
\Rightarrow \sigma_{\omega, n}^{i}=\sigma \cdot \frac{b_{n} \cdot d}{\omega_{n}^{i}}=\sigma \cdot \eta_{n}^{i}, \quad \sigma_{\omega, n}^{o}=\sigma \cdot \frac{b_{n} \cdot d}{\omega_{n}^{o}}=\sigma \cdot \eta_{n}^{o},
\end{gathered}
$$

where $\eta$ is the packing factor that relates the copper area to the total winding area. This factor, already considering all the winding, is different for the inner winding section $\left(\eta^{i}\right)$ and for the outer winding section $\left(\eta^{o}\right)$. Both factors can be calculated as follows:

$$
\eta^{i}=\frac{b \cdot d}{\sum_{n=1}^{m} \omega_{n}^{i}}, \quad \eta^{o}=\frac{b \cdot d}{\sum_{n=1}^{m} \omega_{n}^{o}} .
$$

Therefore, the equivalent conductivity becomes:

$$
\sigma_{\omega}^{i}=\sigma \cdot \eta^{i}, \quad \sigma_{\omega}^{o}=\sigma \cdot \eta^{o} .
$$

Now that the model has reduced the toroidal winding to a one-dimensional problem with an equivalent winding (shown in Fig. 4 (d)), the AC resistance can be obtained through the winding power loss.

\section{AC resistance per unit length in $n t h$ layer}

In order to obtain the power loss per unit length expression for layer $n$, the procedure in [11] is followed: the power flux densities in the inner and outer sides of the layer are computed by means of the Poynting vector $(\boldsymbol{E} x \boldsymbol{H})$ and power loss per layer and unit length is obtained by adding the power flux densities and calculating the real part. The resulting expression can be presented as:

$$
P_{a c, n}=\frac{\Delta \cdot \omega_{n}}{\sigma_{\omega} \cdot d} \cdot\left(\psi_{1}(\Delta) \cdot\left(H_{n, i}-H_{n, o}\right)^{2}+\psi_{2}(\Delta) \cdot 2 \cdot H_{n, i} \cdot H_{n, o}\right),
$$

where $\Delta=d / \delta$, and $\psi_{1}(\Delta)$ and $\psi_{2}(\Delta)$ are functions related to skin and proximity effects [14], respectively, defined as:

$$
\begin{gathered}
\psi_{1}(\Delta)=\frac{\sinh (2 \Delta)+\sin (2 \Delta)}{\cosh (2 \Delta)-\cos (2 \Delta)^{\prime}} \\
\psi_{2}(\Delta)=\frac{\sinh (\Delta)-\sin (\Delta)}{\cosh (\Delta)+\cos (\Delta)} .
\end{gathered}
$$

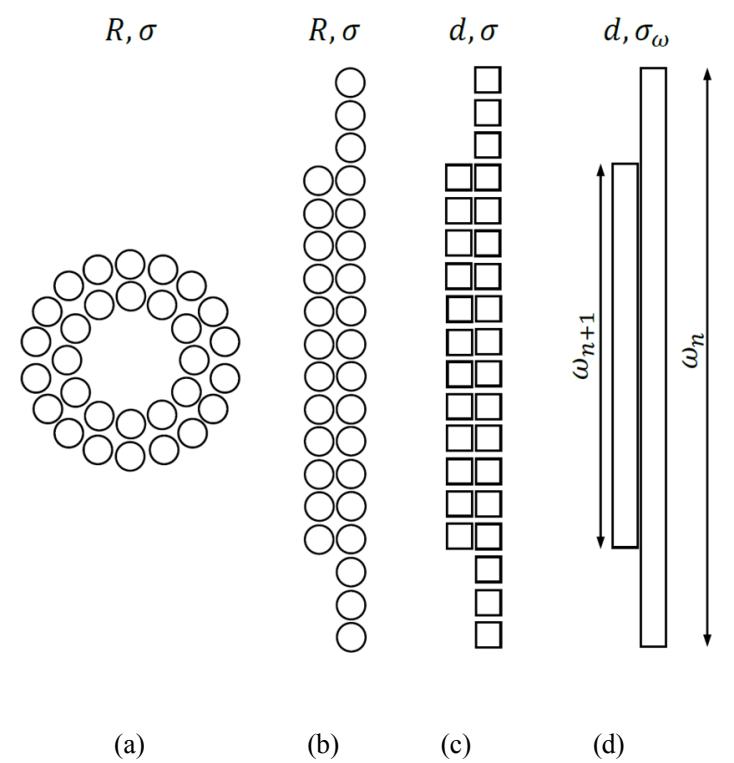

Fig. 4. Round-wire toroidal winding transformations in the proposed model: (a) round-section wires, toroidal or 'doughnut' shape; (b) round-section wires, unfolded; (c) square-section wires, first transformation; (d) equivalent winding (foils), second transformation. 
Regarding the geometrical features of toroidal inductors, $P_{a c, n}$ must be calculated separately for the inner winding section $\left(P_{a c, n}^{i}\right)$ and the outer section $\left(P_{a c, n}^{o}\right)$. The same applies to factor $\Delta$, due to the differences on the packing factor between inner and outer sides, as stated in (13). Therefore, the winding power loss per layer and unit length becomes:

$$
\begin{gathered}
P_{a c, n}^{i}=\frac{\Delta^{\mathrm{i}} \cdot \omega_{n}^{i}}{\sigma_{\omega}^{i} \cdot d}\left(\psi_{1}\left(\Delta^{\mathrm{i}}\right) \cdot\left(H_{n, i}^{i}-H_{n, o}^{i}\right)^{2}+\psi_{2}\left(\Delta^{\mathrm{i}}\right) \cdot 2 H_{n, i}^{i} H_{n, o}^{i}\right), \\
P_{a c, n}^{o}=\frac{\Delta^{o} \cdot \omega_{n}^{o}}{\sigma_{\omega}^{o} \cdot d}\left(\psi_{1}\left(\Delta^{o}\right) \cdot\left(H_{n, i}^{o}-H_{n, o}^{o}\right)^{2}+\psi_{2}\left(\Delta^{o}\right) \cdot 2 H_{n, i}^{o} H_{n, o}^{o}\right) .
\end{gathered}
$$

Then, introducing (10) into (17) and operating, the power loss per unit length in the $n t h$ layer is obtained as:

$$
\begin{gathered}
P_{a c, n}^{i}=\frac{\Delta^{\mathrm{i}} \cdot I^{2}}{\sigma \cdot d^{2}}\left(\psi_{1}\left(\Delta^{\mathrm{i}}\right) \cdot b_{n}+\psi_{2}\left(\Delta^{\mathrm{i}}\right) \cdot 2 \cdot\left(\frac{x_{n, i}^{2}}{b_{n}}+x_{n, i}\right)\right), \\
P_{a c, n}^{o}=\frac{\Delta^{\mathrm{o}} \cdot I^{2}}{\sigma \cdot d^{2}}\left(\psi_{1}\left(\Delta^{\mathrm{o}}\right) \cdot b_{n}+\psi_{2}\left(\Delta^{\mathrm{o}}\right) \cdot 2 \cdot\left(\frac{x_{n, i}^{2}}{b_{n}}+x_{n, i}\right)\right),
\end{gathered}
$$

where $\Delta^{\mathrm{i}}$ and $\Delta^{\mathrm{o}}$ can be expressed as follows:

$$
\Delta^{\mathrm{i}}=\frac{d}{\frac{1}{\sqrt{\pi \cdot \mu_{0} \cdot f \cdot \sigma \cdot \eta^{i}}}}, \quad \Delta^{o}=\frac{d}{\frac{1}{\sqrt{\pi \cdot \mu_{0} \cdot f \cdot \sigma} \cdot \eta^{o}}} .
$$

The relationship between power loss and resistance in the winding can be expressed as:

$$
P_{a c, n}=R_{a c, n} \cdot I^{2} .
$$

Combining (18) and (20), the formula for the AC resistance per layer and unit length in toroidal inductors can be presented as follows:

$$
\begin{aligned}
& R_{a c, n}^{i}=\Delta^{\mathrm{i}} \cdot \frac{1}{\sigma \cdot d^{2}} \cdot\left(\psi_{1}\left(\Delta^{\mathrm{i}}\right) \cdot b_{n}+\psi_{2}\left(\Delta^{\mathrm{i}}\right) \cdot 2 \cdot\left(\frac{x_{n, i}^{2}}{b_{n}}+x_{n, i}\right)\right), \\
& R_{a c, n}^{o}=\Delta^{\mathrm{o}} \cdot \frac{1}{\sigma \cdot d^{2}} \cdot\left(\psi_{1}\left(\Delta^{\mathrm{o}}\right) \cdot b_{n}+\psi_{2}\left(\Delta^{\mathrm{o}}\right) \cdot 2 \cdot\left(\frac{x_{n, i}^{2}}{b_{n}}+x_{n, i}\right)\right) .
\end{aligned}
$$

Then, the total AC resistance per layer and unit length is directly computed as the average of inner and outer $\mathrm{AC}$ resistances as:

$$
R_{a c, n}=\frac{1}{2} \cdot\left(R_{a c, n}^{i}+R_{a c, n}^{o}\right) .
$$

Introducing (21) into (22) and arranging terms, the following formula is obtained:

$$
\begin{aligned}
& R_{a c, n}=\frac{1}{2 \cdot \sigma \cdot d^{2}} \cdot\left[b_{n} \cdot\left(\Delta^{\mathrm{i}} \cdot \psi_{1}\left(\Delta^{\mathrm{i}}\right)+\Delta^{\mathrm{o}} \cdot \psi_{1}\left(\Delta^{\mathrm{o}}\right)\right)+\right. \\
& \left.+2 \cdot\left(x_{n, i}^{2} / b_{n}+x_{n, i}\right) \cdot\left(\Delta^{\mathrm{i}} \cdot \psi_{2}\left(\Delta^{\mathrm{i}}\right)+\Delta^{\mathrm{o}} \cdot \psi_{2}\left(\Delta^{\mathrm{o}}\right)\right)\right] .
\end{aligned}
$$

\section{Proposed resistance factor for toroidal windings, $F_{R}$}

Once the AC resistance per layer and unit length is calculated, it is possible to obtain the winding AC resistance per unit length, $R_{a c}$, by summing $R_{a c, n}$ from $n=1$ to the total number of layers, $m$. Considering the expanded expression in (23), all the factors are independent of $n$, except for the factors multiplying the $\psi_{1}$ and $\psi_{2}$ terms.

Thus, the summation can be applied only to these two factors. First, the summation of the factor multiplying $\psi_{1}$ term, which is $b_{n}$, directly becomes the total number of turns:

$$
\sum_{n=1}^{m} b_{n}=b
$$

Then, the summation of the second factor, being the one that multiplies $\psi_{2}$ term, is defined as $\phi$ and can be formulated in its compact expression as:

$$
\phi=2 \cdot \sum_{n=1}^{m}\left(\frac{x_{n, i}^{2}}{b_{n}}+x_{n, i}\right) .
$$

As a result of (24) and (25), and taking $b$ as a common factor, the winding $\mathrm{AC}$ resistance per unit length can be obtained as:

$$
\begin{aligned}
R_{a c}= & \frac{b}{2 \cdot \sigma \cdot d^{2}} \cdot\left[\Delta^{\mathrm{i}} \cdot \psi_{1}\left(\Delta^{\mathrm{i}}\right)+\Delta^{\mathrm{o}} \cdot \psi_{1}\left(\Delta^{\mathrm{o}}\right)+\right. \\
& \left.+\frac{\phi}{b} \cdot\left(\Delta^{\mathrm{i}} \cdot \psi_{2}\left(\Delta^{\mathrm{i}}\right)+\Delta^{\mathrm{o}} \cdot \psi_{2}\left(\Delta^{\mathrm{o}}\right)\right)\right],
\end{aligned}
$$

where $\phi / b$ is the ratio between (25) and (24), an easy-tocompute factor, unique for round-wire toroidal inductor windings. This factor only depends on geometrical features, particularly on the total number of layers $(m)$ and on factor $A$, previously defined in (4). The expanded form of factor $\phi / b$ can be expressed as follows:

$$
\begin{gathered}
\frac{\phi}{b}=\sum_{k=0}^{m-1}\left(\frac{1}{A-1-2 k}\right)\left[\frac{\left(A^{2}-1\right)^{2}}{8 m(A-m)}+2 m(A-m)-A^{2}+1\right]+ \\
+\frac{m\left(4 \cdot A^{2}-9 \cdot A \cdot m+5 \cdot m^{2}\right)}{8(A-m)}-\frac{1}{8}\left[(A-m)^{2}+3\right] .
\end{gathered}
$$

Taking into account that the resistance factor is the AC to $\mathrm{DC}$ resistance ratio, the DC resistance per unit length must be defined as well:

$$
R_{d c}=\frac{b}{\sigma \cdot d^{2}} .
$$

Finally, dividing (26) by (28), the resistance factor formula for the proposed model results in:

$$
\begin{aligned}
F_{R} & =\frac{1}{2} \cdot\left[\Delta^{\mathrm{i}} \cdot \psi_{1}\left(\Delta^{\mathrm{i}}\right)+\Delta^{\mathrm{o}} \cdot \psi_{1}\left(\Delta^{\mathrm{o}}\right)+\right. \\
& \left.+\frac{\phi}{b} \cdot\left(\Delta^{\mathrm{i}} \cdot \psi_{2}\left(\Delta^{\mathrm{i}}\right)+\Delta^{\mathrm{o}} \cdot \psi_{2}\left(\Delta^{\mathrm{o}}\right)\right)\right] .
\end{aligned}
$$

As can be deduced from (27) and (29), the proposed model is only based on frequency, $f$, and geometrical parameters, such as $m, A, d, \eta$. It is also remarkable that the expression for $F_{R}$ is independent of core permeability. Nevertheless, the inductor must fulfil that there is no concentrated air gap in the core.

\section{STEP-BY-STEP Proposed Model IMPLEMENTATION}

The proposed model achieves a compact, easy-to-use formula for computing the resistance factor of round-wire toroidal inductors. In order to calculate $F_{R}$, the next steps need to be followed:

1) Obtain the basic geomatrical parameters of core $(I D, O D)$ and winding $(2 R)$, as well as the total number of turns $(b)$ and the number of layers $(m)$.

2) Calculate factor $A$ as in (4).

3) Compute the layer heights, $\omega_{n}^{i}$ and $\omega_{n}^{o}$, using (9).

4) Obtain the parameters of equivalent winding: thickness $(d)$ through (11) and inner and outer packing factors $\left(\eta^{i}, \eta^{o}\right)$ through (13). 
5) Compute the frequency-dependent functions. First, apply (19) to obtain $\Delta^{i}$ and $\Delta^{o}$. Then, introduce the results in (16), so functions $\psi_{1}\left(\Delta^{i}\right), \psi_{1}\left(\Delta^{o}\right), \psi_{2}\left(\Delta^{i}\right)$ and $\psi_{2}\left(\Delta^{o}\right)$ are calculated.

6) Knowing $m$ and factor $A$ from steps 1) and 2), respectively, calculate factor $\phi / b$ using (27).

7) Obtain the resistance factor, $F_{R}$, by introducing the results of steps 5) and 6) into (29).

The described process can be easily applied to a given inductor whose characteristics are already known. More interestingly, it can be programmed as well and included in an inductor design algorithm in which different cores, windings and frequencies are to be evaluated. Thus, offering the designer a useful, straightforward tool in the design stage of the toroidal inductors.

\section{VALIDATION}

The proposed model is validated through FEA software FEMM and compared with Dowell's formula. In order to consider realistic designs with a wide variation of factor $A$, including extreme values, four power inductors are designed. The inductors use two different toroidal powder cores from CSC (CH467060 and CH778060) [15] and two different wire gauges (AWG11 and AWG15). As a result, factor $A$ is varied in a range from 10.5 to 34 . The main features of these inductors are presented in Table I (geometrical parameters, number of layers and turns, and rated current assuming 30\% roll-off). The FEMM setup for the simulation of each of the designed inductors is depicted in Fig. 5.

Finite elements simulation, Dowell's formula and the proposed model are applied to the inductors in Table I, computing $F_{R}$ in the frequency range of interest, from $10 \mathrm{~Hz}$ to $200 \mathrm{kHz}$, regarding that in power applications the main harmonic is typically below $100 \mathrm{kHz}$. Then, the results of the resistance factor are represented in Fig. 6 (C467W11 and 467W15) and Fig. 7 (C778W11 and C778W15) versus frequency, for FEMM (red, dots), Dowell (blue, solid) and proposed (black, solid). Moreover, resistance factor at several frequencies of interest are included in Table II, along with the relative deviation of each model when compared to simulation.

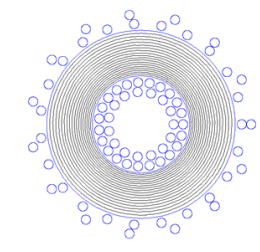

(a)

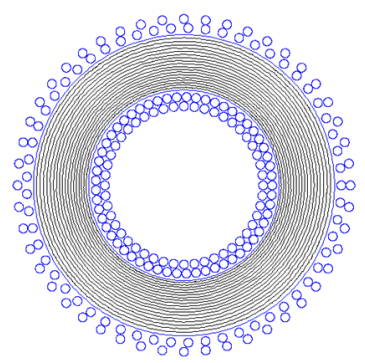

(c)

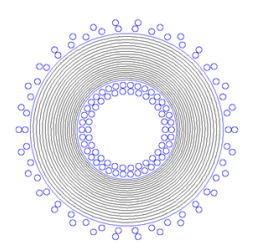

(b)

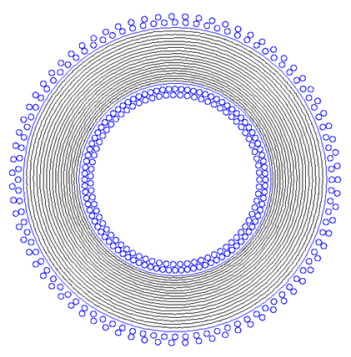

(d)
Fig. 5. Designed inductors in FEMM: (a) C467W11; (b) C467W15; (c) C778W11; (d) C778W15.
TABLE I. DESIGNED INDUCTORS

\begin{tabular}{|c|c|c|c|c|c|c|}
\hline \multirow[b]{2}{*}{ Inductor } & \multicolumn{6}{|c|}{ Features } \\
\hline & $\begin{array}{c}\text { Core } \\
\text { diameter } \\
(\mathrm{mm})\end{array}$ & $\begin{array}{c}\text { Wire } \\
\text { gauge }\end{array}$ & $\boldsymbol{A}$ & $\begin{array}{c}N^{o} \text { of } \\
\text { layers, } \\
m\end{array}$ & $\begin{array}{c}N^{o} \text { of } \\
\text { turns, } \\
\quad b\end{array}$ & $\begin{array}{c}I \\
(A)\end{array}$ \\
\hline C467W11 & \multirow{2}{*}{$\begin{array}{l}I D=24.1 \\
O D=46.7\end{array}$} & AWG11 & 10.5 & 2 & 38 & 28 \\
\hline C467W15 & & AWG15 & 16.6 & 2 & 68 & 16 \\
\hline C778W11 & \multirow{2}{*}{$\begin{array}{l}I D=49.2 \\
O D=77.8\end{array}$} & AWG11 & 21.4 & 2 & 105 & 18 \\
\hline C778W15 & & AWG15 & 34.0 & 2 & 167 & 12 \\
\hline
\end{tabular}

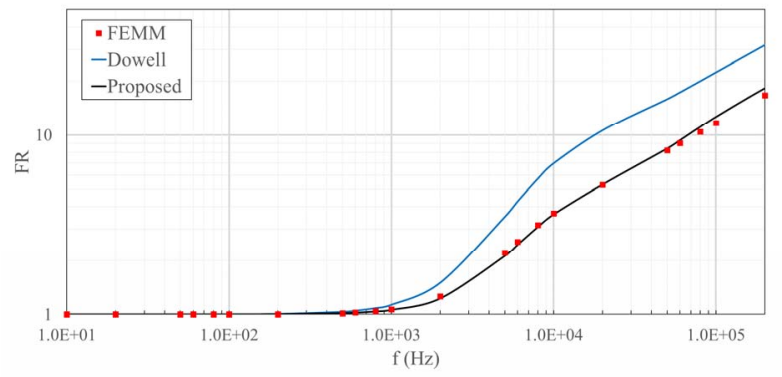

(a)

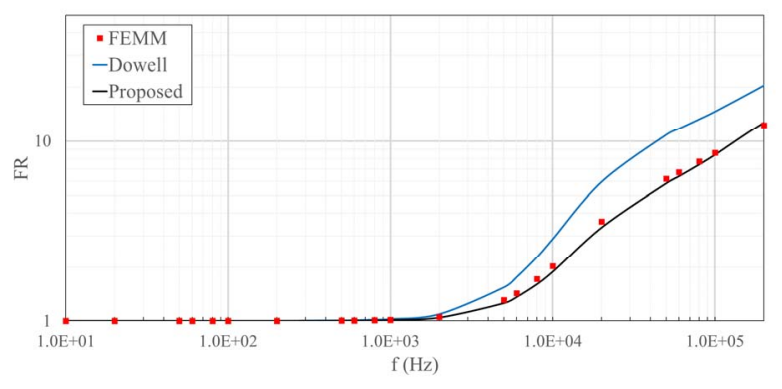

(b)

Fig. 6. Resistance factor $\boldsymbol{F}_{\boldsymbol{R}}$ provided by FEMM, Dowell and proposed model for: (a) C467W11; (b) C467W15.

As can be seen in Fig. 6 and Fig. 7, the proposed model fits better the FEMM results over the entire frequency range. The accuracy of the proposed model is also clear when comparing the maximum deviation: while Dowell's formula provides results with $100 \%$ (C467W11), 75\% (C467W15), 47\% (C778W11) and 45\% (C778W15) maximum relative errors, the proposed model deviates by only $8 \%$ (C467W11), 7\% (C467W15), 13\% (C778W11) and 12\% (C778W15) from FEMM. Hence, it is confirmed that Dowell's formula significantly overestimates the resistance factor when it is applied to toroidal inductors. In addition, it has been demonstrated that the proposed formula is accurate, showing a good agreement with the simulation.

The overestimation detected in Dowell's formula when applied to toroidal inductors is greater as factor $A$ becomes smaller, because then, the packing factor is usually smaller as well, and the 2-D magnetic field is further away from the 1-D distribution that Dowell's formula assumes. Therefore, Dowell's formula provides better results when applied to toroidal inductors with small wire diameter and large core inner diameter. Nevertheless, the proposed model provides better results even in that case. It can also be seen that Dowell's accuracy is strongly dependent on the packing factor, as deduced from the maximum relative deviations. However, the proposed model shows a greater immunity to packing factor variations, keeping close to FEMM results. 


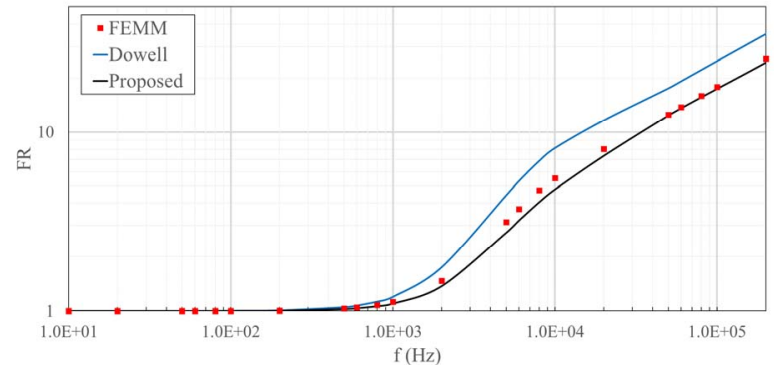

(a)

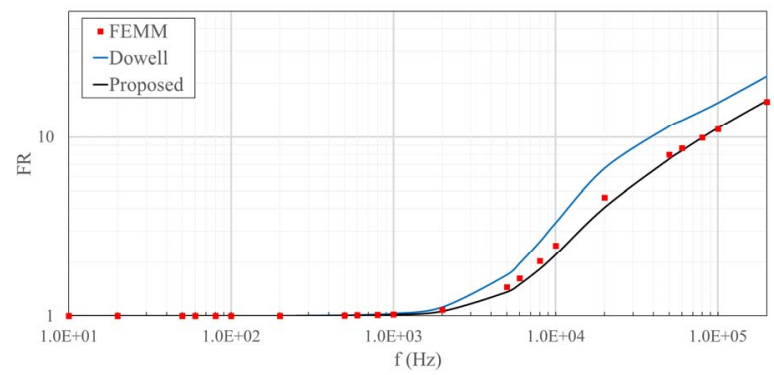

(b)

Fig. 7. Resistance factor $\boldsymbol{F}_{\boldsymbol{R}}$ provided by FEMM, Dowell and proposed model for: (a) C778W11; (b) C778W15.

TABLE II. RESISTANCE FACTOR RESULTS

\begin{tabular}{|c|c|c|c|c|c|}
\hline \multirow[b]{2}{*}{ C467W11 } & \multirow{2}{*}{$\begin{array}{c}\text { FEMM } \\
F_{R} \\
\end{array}$} & \multicolumn{2}{|c|}{ Dowell } & \multicolumn{2}{|c|}{ Proposed } \\
\hline & & $F_{R}$ & $\begin{array}{c}\text { Error } \\
(\%)\end{array}$ & $F_{R}$ & $\begin{array}{c}\text { Error } \\
\text { (\%) }\end{array}$ \\
\hline$f=10 \mathrm{kHz}$ & 3.67 & 6.96 & 89.6 & 3.63 & -1.2 \\
\hline$f=20 \mathrm{kHz}$ & 5.30 & 10.62 & 100.1 & 5.31 & 0.1 \\
\hline$f=50 \mathrm{kHz}$ & 8.24 & 15.88 & 92.8 & 8.41 & 2.1 \\
\hline$f=100 \mathrm{kHz}$ & 11.70 & 22.39 & 91.3 & 12.67 & 8.3 \\
\hline \multirow[b]{2}{*}{ C467W15 } & FEMM & \multicolumn{2}{|c|}{ Dowell } & \multicolumn{2}{|c|}{ Proposed } \\
\hline & $F_{R}$ & $\boldsymbol{F}_{R}$ & $\begin{array}{c}\text { Error } \\
(\%)\end{array}$ & $F_{R}$ & $\begin{array}{c}\text { Error } \\
(\%)\end{array}$ \\
\hline$f=10 \mathrm{kHz}$ & 2.04 & 2.89 & 41.7 & 1.89 & -7.2 \\
\hline$f=20 \mathrm{kHz}$ & 3.60 & 5.96 & 65.3 & 3.34 & -7.4 \\
\hline$f=50 \mathrm{kHz}$ & 6.18 & 10.83 & 75.1 & 5.84 & -5.6 \\
\hline$f=100 \mathrm{kHz}$ & 8.62 & 14.62 & 69.6 & 8.40 & -2.5 \\
\hline \multirow[b]{2}{*}{ C778W11 } & FEMM & \multicolumn{2}{|c|}{ Dowell } & \multicolumn{2}{|c|}{ Proposed } \\
\hline & $F_{R}$ & $\boldsymbol{F}_{R}$ & $\begin{array}{c}\text { Error } \\
(\%)\end{array}$ & $F_{R}$ & $\begin{array}{c}\text { Error } \\
(\%)\end{array}$ \\
\hline$f=10 \mathrm{kHz}$ & 5.53 & 8.15 & 47.4 & 4.77 & -13.1 \\
\hline$f=20 \mathrm{kHz}$ & 8.05 & 11.67 & 45.0 & 7.36 & -8.6 \\
\hline$f=50 \mathrm{kHz}$ & 12.56 & 17.60 & 40.2 & 12.40 & -1.3 \\
\hline$f=100 \mathrm{kHz}$ & 17.94 & 24.94 & 39.0 & 17.49 & -2.6 \\
\hline \multirow[b]{2}{*}{ C778W15 } & FEMM & \multicolumn{2}{|c|}{ Dowell } & \multicolumn{2}{|c|}{ Proposed } \\
\hline & $F_{R}$ & $F_{R}$ & $\begin{array}{c}\text { Error } \\
(\%)\end{array}$ & $F_{R}$ & $\begin{array}{c}\text { Error } \\
(\%)\end{array}$ \\
\hline$f=10 \mathrm{kHz}$ & 2.49 & 3.34 & 34.3 & 2.21 & -10.9 \\
\hline$f=20 \mathrm{kHz}$ & 4.62 & 6.70 & 45.0 & 4.06 & -12.0 \\
\hline$f=50 \mathrm{kHz}$ & 7.95 & 11.48 & 44.5 & 7.55 & -5.0 \\
\hline$f=100 \mathrm{kHz}$ & 11.08 & 15.54 & 40.2 & 11.26 & 1.6 \\
\hline
\end{tabular}

\section{CONCLUSIONS}

Low-permeability toroidal inductors are present in many power electronic applications. Thus, designers need a flexible, accurate tool to calculate high-frequency winding loss and, this way, be able to obtain a cost and volume optimal design. Regarding the available options, although FEA software is accurate, it is not flexible, and becomes impractical when many designs and operating frequencies are simulated. The other existing alternative, analytical models, such as Dowell's, have been proved not to be suitable for toroidal inductor windings.

This paper presents an analytical model that takes into account the particularities of ungapped round-wire toroidal inductors, resulting in an accurate, straightforward formula. The ease of use of the proposed model is demonstrated by means of a step-by-step implementation. Therefore, this formula allows the designer to assess many different designs and operating frequencies in a very short period of time. Finally, the proposed model is validated through FEA software for four power toroidal inductors and compared with the results provided by Dowell's formula. The results show that, while Dowell's formula incurs an unacceptable overestimation, the proposed model is in good agreement with the simulation, demonstrating its accuracy.

\section{REFERENCES}

[1] J. Imaoka, K. Okamoto, M. Shoyama, Y. Ishikura, M. Noah, and M. Yamamoto, "Modeling, magnetic design, simulation methods, and experimental evaluation of various powder cores used in power converters considering their dc superimposition characteristics," IEEE Trans. Power Electron., vol. 34, no. 9, pp. 9033-9051, 2019.

[2] R. P. Wojda and M. K. Kazimierczuk, "Winding Resistance and Power Loss of Inductors with Litz and Solid-Round Wires," IEEE Trans. Ind. Appl., vol. 54, no. 4, pp. 3548-3557, 2018.

[3] F. Forest, E. Labouré, T. A. Meynard, and V. Smet, "Design and comparison of inductors and intercell transformers for filtering of PWM inverter output," IEEE Trans. Power Electron., vol. 24, no. 3, pp. 812-821, 2009.

[4] M. R. Khowja et al., "Novel Motor-Shaped Rotational Inductor for Motor Drive Applications," IEEE Trans. Ind. Electron., vol. 67, no. 3, pp. 1844-1854, 2020.

[5] R. A. Torres and T. M. Jahns, "Design of High-Performance Toroidal DC-link Inductor for Current-Source Inverters," 2019 IEEE Appl. Power Electron. Conf. Expo., pp. 2694-2701, 2019.

[6] J. Qiu, A. J. Hanson, and C. R. Sullivan, "Design of toroidal inductors with multiple parallel foil windings," 2013 IEEE 14th Work. Control Model. Power Electron. COMPEL 2013, pp. 1-6, 2013.

[7] B. Liu, R. Ren, F. Wang, D. Costinett, and Z. Zhang, "Winding Scheme with Fractional Layer for Differential-Mode Toroidal Inductor," IEEE Trans. Ind. Electron., vol. 67, no. 2, pp. 1592-1604, 2020.

[8] U. Patil and N. Harischandrappa, "Analysis and Design of a HighFrequency Isolated Full-Bridge ZVT CLL Resonant DC-DC Converter," IEEE Trans. Ind. Appl., vol. 55, no. 5, pp. 4993-5004, 2019.

[9] M. S. Rylko, B. J. Lyons, J. G. Hayes, and M. G. Egan, "Revised magnetics performance factors and experimental comparison of highflux materials for high-current dc-dc inductors," IEEE Trans. Power Electron., vol. 26, no. 8, pp. 2112-2126, 2011.

[10] P. L. Dowell, "Effects of eddy currents in transformer windings," Proc. Inst. Electr. Eng., vol. 113, no. 8, p. 1387, 1966.

[11] W. G. Hurley, E. Gath, and J. G. Breslin, "Optimizing the AC resistance of multilayer transformer windings with arbitrary current waveforms," IEEE Trans. Power Electron., vol. 15, no. 2, pp. 369-376, 2000.

[12] A. Reatti and M. K. Kazimierczuk, "Comparison of various methods for calculating the AC resistance of inductors," IEEE Trans. Magn., vol. 38, no. 3, pp. 1512-1518, 2002.

[13] E. L. Barrios, A. Ursua, L. Marroyo, and P. Sanchis, "Analytical Winding Loss Calculation for High-Frequency Low-Permeability Inductors," 2017 IEEE 18th Work. Control Model. Power Electron., pp. 1-7, 2017.

[14] J. A. Ferreira, "Improved Analytical Modeling of Conductive Losses in Magnetic Components," IEEE Trans. Power Electron., vol. 9, no. 1, pp. 127-131, 1994.

[15] Toroidal Magnetic Powder Cores, Changsung Corporation (CSC). [Online]. Available: www.changsung.com. 